\title{
Optical configuration for a micro-arcsecond astrometric interferometer in space
}

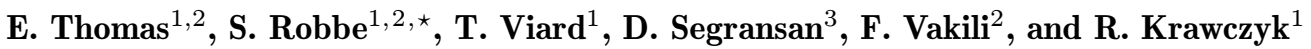 \\ 1 Alcatel Space Industries, Future Projects, 100 Bd. du Midi, 06322 Cannes la Bocca Cedex, France \\ 2 Observatoire de la Côte d'Azur, Département Fresnel, UMR 6528, Av. Copernic, 06130 Grasse, France \\ 3 Observatoire de Grenoble, Laboratoire d'Astrophysique, 414 rue de la Piscine, Domaine Universitaire St Martin d'Hères, \\ BP. 53, 38041 Grenoble Cedex 9, France
}

Received January 26, 1998; accepted April 30, 1999

\begin{abstract}
One of the ESA candidate cornerstone missions is dedicated to wide-angle astrometry and aims at dramatically increasing the accuracy of astrometric measurements and the limiting magnitude with respect to Hipparcos (ESA Symposium, Venice, 1997). This mission was initially designated by the acronym GAIA (Global Astrometric Interferometer for Astrophysics) and featured several Fizeau interferometers. One important drawback of the original configuration proposed by Lindegren \& Perryman (1996) was the confusion effect. This effect, which is a superposition of the light coming from different objects, is due to recording the fringe signal in the exit pupil plane of the instrument, necessary to relax the pixel size in the considered configuration. In order to overcome the confusion effect while retaining the original Fizeau interferometer concept, we present an optical configuration allowing direct fringe detection, i.e. in the image plane. It features focal length of $30 \mathrm{~m}$ inducing technologically feasible pixel size in the bandwidth of interest, and meets the condition to fit the Ariane $\mathrm{V}$ launcher fairing.
\end{abstract}

Key words: astrometry — instrumentation: detectors instrumentation: interferometers — methods: analytical — methods: numerical — techniques: interferometric

\section{Introduction}

The success of Hipparcos (Mignard 1995; van Leeuwen 1997; Kovalevsky 1998) demonstrated the feasibility of

Send offprint requests to: E. Thomas

* Present address: Université de Nice Sophia Antipolis, Laboratoire d'Astrophysique, 28 Av. Valrose, 06108 Nice Cedex 2, France.

Correspondence to: ethomas@obs-azur.fr obtaining astrometric measurements in space with milliarcsecond accuracy. The result is the construction of a catalogue with 120000 stars as faint as the visual 12th magnitude (Perryman 1997).

The mission initially called Global Astrometric Interferometer for Astrophysics (GAIA, Lindegren \& Perryman 1996), submitted to ESA for its Horizon $2000+$ programme as a candidate cornerstone mission, is a successor of Hipparcos. Its goal was to bring a breakthrough in global astrometric observations with extremely high accuracy, down to $10 \mu$ as, with a limiting visual magnitude of 15-16. Later studies (Gilmore et al. 1998) have lead to an improved design which allows observation (at a reduced accuracy) to significantly fainter magnitudes. In the following, we considered as work hypothesis an achievable $10 \mu$ as astrometric accuracy for a 15 th magnitude star.

GAIA will provide multi-band, multi-epoch photometry. The mission life-time is expected to be 5 years at least, necessary to attain the desired astrometric accuracy with a considerable amount of by-product results such as a significant survey of extra-solar planets and test of the general relativity theory (Lindegren \& Perryman 1996; Perryman et al. 1997; Lattanzi et al. 1997). With $10 \mu$ as astrometry and a 15th magnitude limit, GAIA's parallax horizon will envelope the Milky-way Galaxy, its halo, many globular clusters, reaching very probably the Magellanic Clouds. Among other targets, GAIA will also determine the distance of all galactic Cepheid variables, RR Lyrae and long period pulsators like Miras and will refine at an unrivalled level, the Period - Luminosity - Metallicity relation. The impact of such observations on cosmology is immense but GAIA will certainly bring news that we are far from suspecting at this time.

In order to perform global astrometry, a large number of objects, uniformly distributed on the celestial sphere, 
should be observed. To achieve the construction of a distortionless reference system over the whole sky from large angle measurements, a sky scanning law similar to that of Hipparcos will be used. As it spins about an axis, the satellite sees, over one revolution, all the stars present over a great circle. Measuring angular deviations yields these stars' positions in mutual relations. Each circle is itself linked to all other in a final data processing step, thereby graduating from one-dimensional to two-dimensional information on position. Thus, a network is built during the mission, that binds together all of the observed objects, yielding both a reference frame and the positions, proper motions and parallaxes of the objects in that reference frame at the end of the mission.

As far as simultaneous observations are needed in several directions, the instrumental concept proposed by Lindegren \& Perryman (1996) is composed of three interferometers whose lines of sight are separated by constant angles $54^{\circ}, 78.5^{\circ}$, and $132.5^{\circ}$. Although only two instruments are sufficient to fulfil the mission, the third one insures a redundancy in the measurements.

The associated optical configuration was analysed by (Loiseau \& Shaklen 1996). The choice of a Fizeau interferometer (versus a Michelson configuration) was imposed by the necessity to observe with a large co-phased field of view (the word "co-phased" being used to describe a field of view in which interference fringes are not degraded by aberrations). The entrance pupil diameter was $0.55 \mathrm{~m}$, the baseline $2.45 \mathrm{~m}$, and the equivalent focal length $11.55 \mathrm{~m}$. The latter, combined with a $1 \mathrm{deg}^{2}$ field of view yields a $20 \times 20 \mathrm{~cm}^{2}$ focal plane. In such a configuration, the fringe period is $2.6 \mu \mathrm{m}$ (at $\lambda=550 \mathrm{~nm}$ ) and the Airy disk diameter $28 \mu \mathrm{m}$, requiring a pixel size of $1.3 \times 28 \mu \mathrm{m}^{2}$ (maximum size imposed by Shannon criterion), technologically unfeasible as yet. To overcome the pixel size problem, a solution was proposed for the fringe detection consisting in dividing the whole field of view in subfields and linking them with a modulating grid. The observation is recorded in the pupil plane. The spatial modulation of the intensity due to interference fringes in the image plane is transformed into a temporal modulation detected on a mono-pixel detector in the pupil plane. The main drawback of this solution is the confusion effect: the infalling fluxes of different stars within the same subfield cannot be distinguished. One of us (Thomas 1996) evaluated quantitatively the importance of this effect, showing the degradation induced on the signal phase measurement by observing in crowded fields.

To overcome the confusion effect, we propose a new optical configuration (Gai et al. 1997; Thomas et al. 1997) suitable to an astrometric mission such as GAIA, which would enable direct observations in the image focal plane (direct fringe detection), taking into account the expected state-of-the-art CCD technologies. This work was the target of a study conducted under ALENIA's prime contractorship in 1997 (in the framework of the APLTAMTS ESA contract). Other concepts for GAIA presently exist and are under study. In particular, a Concept and Technology Study performed under a different ESA contract has lead to a rather different (non-interferometric) design, which is currently the baseline version of GAIA considered by ESA. Our study presents an alternative solution, which combines the original concept of a Fizeau interferometer with a technologically reasonable solution for direct fringe detection.

In the following, we derive the requirements of the direct fringe detection configuration (field of view, focal plane, telescope accommodation, calibration and geometrical stability). We present in detail our optical configuration in Sect. 2, as well as the foreseeable CCD technology. We also present the optical quality in terms of rms spot diagram diameter. In Sect. 3, we give the optical configuration performance in terms of aberrations and mechanical tolerances. A way to assess the performance in terms of estimated fringe contrast was implemented using Fourier optics on a Software for Interferometric Visibility Analysis (SIVA), an algorithm developed at Alcatel Space Industries, Cannes.

\section{Configuration for direct fringe detection}

In this section, we give the requirements for deriving the optical configuration: estimated field of view, focal plane characteristics, instrument accommodation in the Ariane $\mathrm{V}$ fairing, optical quality and the sensitivity to misalignments.

The optical concept derived from these considerations is presented, as well as a possible associated focal plane and its characteristics according to available CCD Philips Imaging Technology's expertise.

\subsection{Requirements}

\subsubsection{Field of view requirements}

The major requirements to specify an optical configuration suitable for an astrometric mission come from the astrometric accuracy and the limiting magnitude imposed by the scientific objectives to be fulfilled by the mission.

The analytical relationships between the various observational parameters are provided by equations in Lindegren \& Perryman (1996).

Assuming other parameters to be known, it can be shown that the requirement to achieve $10 \mu$ as accuracy for a visual 15th magnitude star will give the minimum dimension of the field of view (FOV) of each interferometer. 


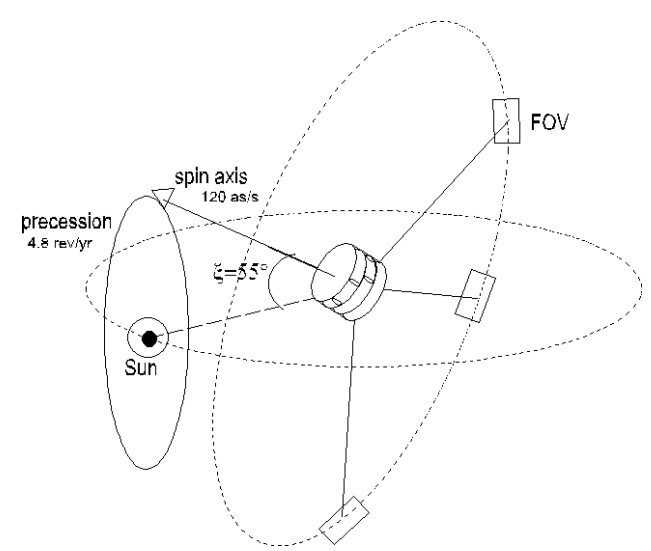

Fig. 1. The sky scanning law. The satellite is spinning at a constant rate and the spin axis revolves about the direction of the Sun

This is not the only requirement for specifying the interferometric FOV. The required sky coverage, and, in particular, the partial overlap between the sky strips covered by the FOV on successive great circles, will be achieved only if the across-scan dimension of the FOV is larger than the angular separation between two great circles. The latter depends on the spin rate of the spacecraft, the solar aspect angle, and the precession velocity $V_{\mathrm{p}}$ of the spin axis. Typical values (Fig. 1) for the spin rate and solar aspect angle are respectively $120 \mathrm{as} / \mathrm{s}$ and $55^{\circ}$ (Lindegren $\&$ Perryman 1996). For the precession velocity, it is deduced from the preceeding parameters, i.e. 4.8 revolutions per year. Given these values, it is possible to derive the displacement of the spin axis $D_{\mathrm{s}}$, after one revolution, (i.e, the angle between the spin axis at time $t_{1}$ and at time $\left.t_{1}+\Delta t\right)$ :

$D_{\mathrm{s}}=V_{\mathrm{p}} \cdot \Delta t \cdot \sin (\xi)$

where $\Delta t$ is the rotational period for the satellite. In this formula the contribution from the annual motion is neglected. From the preceeding values, $D_{\mathrm{s}}=0.48^{\circ}$. In order to achieve a good overlap by subsequent scans, a field size of $0.8^{\circ}$ was chosen (cf. Table 1 ).

\subsubsection{Focal plane assembly}

The requirements on the focal plane are also derived from the mission's scientific objectives. The limiting magnitude and the brightest stars define the electronic dynamic range requirements of the pixels. The spin rate of the spacecraft defines the velocity at which fringes cross the focal plane, on which the instantaneous integration time directly depends. In this section, we translate these requirements in terms of integration time and number of lines on the detector that will have to be summed to reach a sufficient signal to noise ratio during the fringes displacement (TDI mode).
The instantaneous integration time $T_{\mathrm{i}}$ is derived from the along-scan size $\Delta x$ of the pixel and the fringe motion $v$. The parameter $v$ depends on the spin rate $\omega$ of the satellite given in rad/s and on the focal length $F$ of the interferometer given in metres. $T_{\mathrm{i}}$ is then given by:

$T_{\mathrm{i}}=\frac{\Delta x}{v}=\frac{\Delta x}{F \cdot \omega}$.

The number of TDI lines $\left(N_{\text {TDI }}\right)$ required to obtain an acceptable value of the Signal to Noise Ratio $(R)$ can also be estimated. If $r$ is the read out noise of the CCD, the required number of photo-electrons can be written as:

$N_{\mathrm{p}} \approx \frac{R^{2}+\sqrt{R^{4}+4 r^{2} R^{2}}}{2}$.

Given $N_{\mathrm{s}}$, the number of photo-electrons falling from the star per integration time $T_{\mathrm{i}}$, and contributing to a given fringe pattern, we can assume that the central fringe contains $N_{\mathrm{s}} / 5$ from $B / D$ ratio. Hence, if the number of pixels per fringe is given by $P$, a pixel in the central fringe collects:

$N_{\mathrm{c}} \approx \frac{N_{\mathrm{s}}}{5 P}$

photo-electrons. The required number of TDI lines is then given by:

$N_{\mathrm{TDI}} \approx \frac{N_{\mathrm{p}}}{N_{\mathrm{c}}}$

The availability of suitable detectors for fringe detection has to be considered, in terms of:

- pixel size: rectangular pixels with $2.4 \mu \mathrm{m}$ size in the scan direction seem to be achievable according to CCD Philips Imaging Technology's expertise (Peek et al. 1993; Peek et al. 1996);

- sampling rate: a minimum of about $P=3$ pixels per fringe period provides nearly theoretical performance for the fringe location process (the Shannon criterion being a minimum theoretical limit for an ideal signal). Considering an achievable pixel size $\Delta x$ equal to $2.4 \mu \mathrm{m}$, a baseline $B$ of $2.55 \mathrm{~m}$, and a focal length of $30 \mathrm{~m}$, we obtain 2.7 pixels per fringe at $0.55 \mu \mathrm{m}$, which is close to the theoretical value for the fringe location process.

- Achievable quantum efficiency;

- focal plane dimensions: we considered a few tens of centimetres in linear size, typically $40 \mathrm{~cm}$, as a reasonable size.

Taking into account the scan velocity of the satellite, the available integration time for a $2.4 \mu \mathrm{m}$ detector is $133 \mu \mathrm{s}$ at the most. The number of TDI steps compatible with the required signal to noise ratio $(R>3)$, for a 15th visual magnitude star, is then about 1500 (with the hypotheses of a $5 \mathrm{e}^{-}$noise level, $30 \%$ quantum efficiency and $100 \mathrm{~nm}$ bandwidth at $550 \mathrm{~nm}$ ). 
Table 1. Characteristics of the interferometer and focal plane assembly. Detector characteristics are our assumed values as projected from current Philips technology

\begin{tabular}{ll} 
Effective focal length & $30 \mathrm{~m}$ \\
Baseline & $2.55 \mathrm{~m}$ \\
Aperture diameter & $600 \mathrm{~mm}$ \\
Square field of view & $0.8^{\circ} \times 0.8^{\circ}$ \\
Diffraction-limited circular FOV & $1.1^{\circ}$ \\
Fringe width & $6.5 \mu \mathrm{m}$ at $\lambda=550 \mathrm{~nm}$ \\
Airy disk diameter & about $70 \mu \mathrm{m}$ at $\lambda=550 \mathrm{~nm}$ \\
Number of reflexions & $5(3$ aspherical and 2 flat mirrors $)$ \\
Focal plane dimensions & $42 \mathrm{~cm} \times 42 \mathrm{~cm}$ \\
Quantum efficiency & $30 \%$ at $\lambda=550 \mathrm{~nm}$ \\
Number of CCD chips & $4 \times 5$ \\
CCD chip dimensions & $88 \times 110 \mathrm{~mm}^{2}$ \\
Number of $2.4 \mu \mathrm{m}$ per fringe & $2.7 \mathrm{pixels} \mathrm{at} \lambda=550 \mathrm{~nm}$ \\
Total pixel number $(2.4 \mu \mathrm{m} \times 70 \mu \mathrm{m})$ & $175000 \times 6000$ \\
CTE loss & $10^{-7}$ \\
Focal plane temperature & $-50^{\circ} \mathrm{C}$ \\
Read out noise & $5 \mathrm{e}^{-}$ \\
Data rate & $2.8 \mathrm{Gbits} / \mathrm{s}$ \\
\hline
\end{tabular}

\subsubsection{Telescope accommodation requirements}

For an assumed launch with the European launcher Ariane $\mathrm{V}$, the volume of each telescope (in terms of diameter and height of its envelope) is limited by the internal dimensions of Ariane V Speltra fairing $(\phi=4.57 \mathrm{~m}, h=4.85 \mathrm{~m}$ for the cylindrical section) and by the number of instruments to be accommodated on the Payload Module (PLM). Assuming for the service module a height of $1.7 \mathrm{~m}$, the PLM height cannot exceed, say $3 \mathrm{~m}$ (cylindrical section only). This limits the height available for each instrument (three have to be stacked on top of each other). An initial value for each interferometer can be taken as $0.7 \mathrm{~m}$ (internal height of the telescope compartment). This leaves enough room for the accommodation of an instrument as the Auxiliary Radial-Velocity Instrument (ARVI), (Favata \& Perryman 1997), for which, however, the non-cylindrical upper region of the Speltra could be exploited (Fig. 2). The Speltra also limits the diameter of each interferometer. Considering a reasonable thickness of the PLM wall and its thermal cover, the maximum allowable diameter for each interferometer will be about $4.3 \mathrm{~m}$.

Inside the PLM, each telescope shall be mounted with its baseline vector orthogonal to the nominal spin axis of the spacecraft, and with the line of sight oriented according to the values defined for the basic line-of-sight angles.

\subsubsection{Calibration and stability requirements}

Calibration of residual aberrations has to respect the required value of fringe contrast and displacement of the Airy disk centroid, over the whole field of view. An interferometer can be considered diffraction-limited when

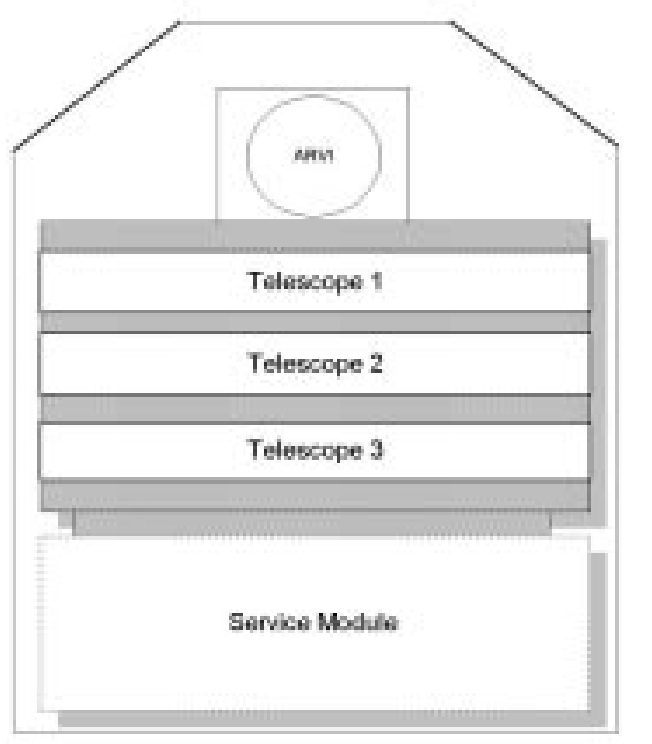

Fig. 2. Telescope accomodation in the Ariane V Speltra fairing

the fringe visibility is at least 0.8 (Cecconi et al. 1997a). Particular attention is paid to the distortion introduced by the optical design, as its level must be compatible with a single TDI clocking rate applicable throughout the FOV (or at least to a single CCD chip).

The distortion introduces a loss of contrast due to the difference between the fringe pattern velocity and the charge transfer.

The evaluation of this effect is done using the desynchronization Modulation Transfer Function evaluated 
by considering that the fringes are equivalent to the modulation signal obtained using a grid on a single pupil. This MTF is given by the following expression:

$M(\mu)=\frac{\sin \left(N_{\mathrm{TDI}} \pi \mu\left(\Delta x-v T_{\mathrm{i}}\right)\right)}{N_{\mathrm{TDI}} \sin \left(\pi \mu\left(\Delta x-v T_{\mathrm{i}}\right)\right)}$

where $v$ is the component of the diffraction pattern velocity in the along-scan direction, $T_{\mathrm{i}}$ the instantaneous integration time, $\Delta x$ the pixel size, $N_{\text {TDI }}$ the number of TDI lines, $\mu$ the spatial frequency.

For this mission, we take $v$ equal to $\omega F$, where $\omega$ is the spin velocity and $F$ is the focal length. For the distortion at any given point within the field of view of the instrument, $F$ becomes $F+\Delta F$. Therefore, there is a difference between the diffraction pattern velocity and the charge transfer one. The latter is given by $\Delta x / T_{\mathrm{i}}=\omega F$. The expression of the desynchronization MTF becomes:

$M(\mu)=\frac{\sin \left(N_{\mathrm{TDI}} \pi \mu \Delta x \frac{\Delta F}{F}\right)}{N_{\mathrm{TDI}} \sin \left(\pi \mu \Delta x \frac{\Delta F}{F}\right)}$.

Each fringe is sampled over 3 pixels, giving the frequency $\mu$ equal to $1 / 3 \Delta x$. $N_{\text {TDI }}$ is equal to 1500 lines (Sect. 2.1.2).

The degradation budget according to the defined independent sources of error must fall below $20 \%$. The following five contributors to the contrast loss were assumed (Cecconi et al. 1997b):

- fringe polychromaticity;

- deviations of the optical system from its nominal configuration;

- jitter of the line of sight;

- TDI desynchronization due to the imperfect knowledge of the scan velocity;

- TDI desynchronization due to distortions.

Assuming that the sources of error contribute equally to the total degradation, the tolerance on fringe contrast for a $9 \%$ degradation is given by:

$\frac{\sin \left(1500 \frac{\pi}{3} \frac{\Delta F}{F}\right)}{1500 \cdot \sin \left(\frac{\pi}{3} \frac{\Delta F}{F}\right)} \geq 0.91$.

This leads to the requirement in terms of distortion:

$\frac{\Delta F}{F} \leq 4.710^{-4}$.

The requirements on opto-mechanical tolerances, in terms of tilt and decenterings, have to be given for each mirror, in order to reach the desired performance in terms of Airy disk location. An instantaneous astrometric accuracy of $100 \mu$ as is required in order to achieve the final $10 \mu \mathrm{as}$ accuracy on the measurements (estimated from Hipparcos results). Considering the 30-m focal length, this accuracy can be achieved if the centroid displacement induced by spurious effects is less than $15 \mathrm{~nm}$.

Alcatel Space Industries has developped an algorithm called SIVA. Dedicated to fringe contrast computation,
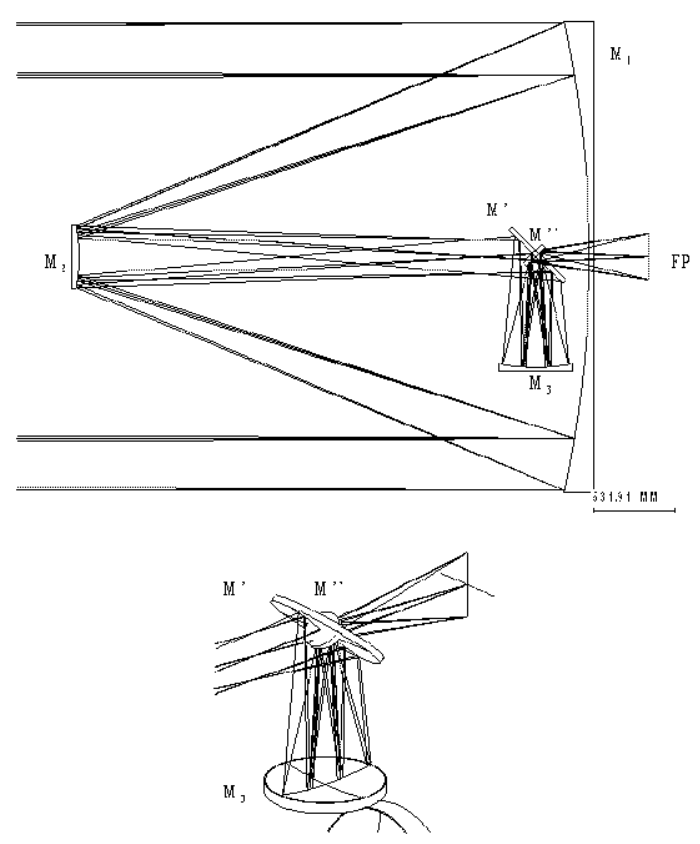

Fig. 3. Korsch configuration and associated relay system

this algorithm is based on Zernike polynomials derived from residual wavefront abberations calculated by $\mathrm{CodeV}$ (a registred product of Optical Reasearch Associates). Opto-mechanical tolerances are directly derived from CodeV.

\subsection{Optical configuration description}

Figure 3 shows the scheme of the proposed optical configuration, which is a Korsch configuration, composed of three aspherical mirrors $\left(M_{1}, M_{2}\right.$ and $\left.M_{3}\right)$. It allows the formation of a real exit pupil, making possible the insertion of both active optics and baffling.

An optical relay system is implemented, allowing to take into account dimensions of the ArianeV envelope and space dedicated to structure and mechanics. This relay system is the combination of two flat mirrors $\left(M^{\prime}\right.$ and $\left.M^{\prime \prime}\right)$, as shown in Fig. 3 .

The fraction of the obstructed field of view due to the optical relay system is about $0.15 \%$. Table 2 gives the optical parameters of the configuration, in terms of mirror curvature radius, conical constants and the distance of the mirrors to the following surface. The rms spot diagrams given by $\mathrm{CodeV}$ using two $60 \mathrm{~cm}$ apertures are given in Fig. 4.

\section{Performance: Optical quality and sensitivity}

In this section, the computation of the performance of the optical configuration is presented in terms of optical 
Table 2. Optical parameters of the Korsch configuration

\begin{tabular}{cccccc}
\hline Surface & $\begin{array}{c}\text { Radius of } \\
\text { curvature }(\mathrm{mm})\end{array}$ & $\begin{array}{c}\text { Conical } \\
\text { constant }\end{array}$ & $\begin{array}{c}4 \text { th } \\
\text { order term }\end{array}$ & $\begin{array}{c}6 \text { th } \\
\text { order term }\end{array}$ & $\begin{array}{c}\text { Distance to the } \\
\text { following surface }\end{array}$ \\
\hline$M_{1}$ & -7654.49 & -0.991257 & $-0.121710^{-14}$ & $0.609810^{-24}$ & -3370.42 \\
$M_{2}$ & -1103.27 & -1.787285 & $0.310410^{-11}$ & $-0.168610^{-17}$ & 3017.73 \\
$M^{\prime}$ & $\infty$ & - & - & - & -747.02 \\
$M_{3}$ & 1270.36 & -1.502707 & $0.543510^{-10}$ & $-0.252410^{-17}$ & 747.02 \\
$M^{\prime \prime}$ & & - & - & - & -743.86 \\
$\mathrm{FP}$ & $\infty$ & - & - & - & 0.0152 \\
\hline
\end{tabular}

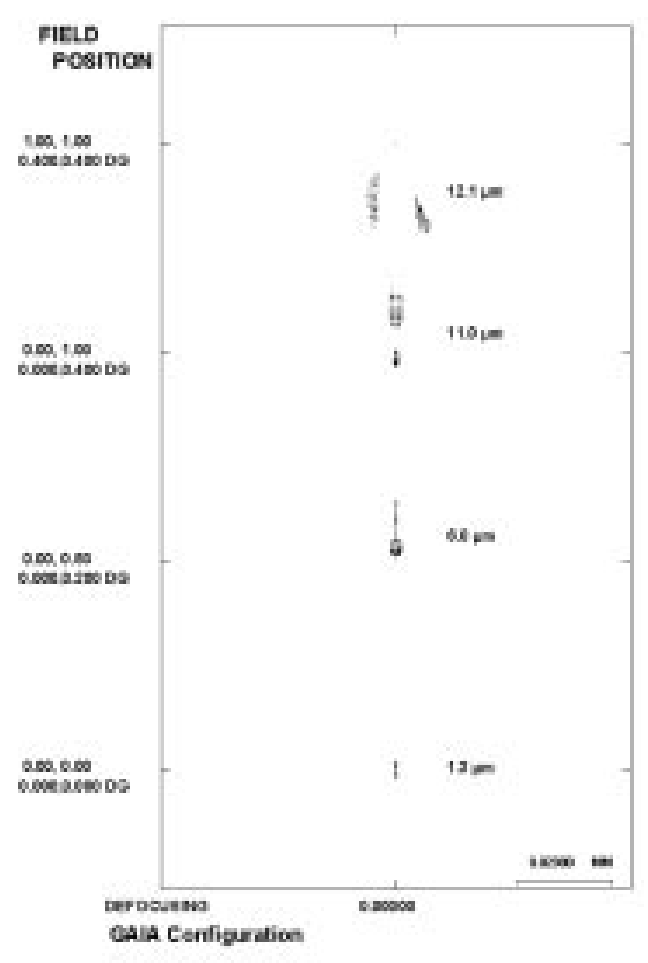

Fig. 4. Spot diagrams for the Korsch configuration with two apertures as for the interferometer

quality and sensitivity to misalignments. The calculations for optical quality were brought together in a dedicated software called SIVA. Results are given in terms of contrast estimation and displacement of the centroid of the point spread function in the field of view. In addition, sensitivity to misalignments (tilt and decenterings) is performed with CodeV.

\subsection{Computation of the optical quality}

We developed an algorithm, called SIVA (Software for Interferometer Visibility Analysis), dedicated to assess the performance of optical configurations for a 2-aperture interferometer. The two identified criteria to evaluate the optical quality are:
- fringe contrast;

- displacement of the Airy disk.

SIVA estimates the fringe contrast from the wavefront decomposition in Zernike polynomials. The coefficients of the Zernike polynomials are given by $\mathrm{CodeV}$ which calculates them for different points in the field of view.

To compute the equations, the main hypotheses are a monochromatic observation and an unresolved star. An aberrated wavefront is expressed in the 2-aperture interferometer plane by the following equation:

$$
\begin{aligned}
& \Psi(u, v)=\left[\Pi\left(\frac{f}{f_{\mathrm{c}}}\right) \otimes \delta\left(u, v+\frac{f_{\mathrm{ci}}}{2}\right)\right] \cdot h_{1}(u, v)+ \\
& {\left[\Pi\left(\frac{f}{f_{\mathrm{c}}}\right) \otimes \delta\left(u, v-\frac{f_{\mathrm{ci}}}{2}\right)\right] \cdot h_{2}(u, v)}
\end{aligned}
$$

where the symbols are:

- $f$ is equal to $\sqrt{u^{2}+v^{2}}, u$ and $v$ being the spatial frequencies;

- $f_{\mathrm{c}}$ is equal to $D / \lambda F, D$ being the pupil diameter and $F$ the instrument's focal length;

- $f_{\text {ci }}$ is equal to $B / \lambda F, B$ being the baseline;

- $\Pi$ is the pupil function defined as $\Pi=0$ for $f / f_{\mathrm{c}}>1$ and $=1$ elsewhere;

- $\otimes$ is the convolution operator;

- $\delta$ is the Dirac's function;

- $h_{i}$ is equal to $\exp \left(j \cdot \Delta_{i}(u, v)\right)$, where $\Delta_{i}(u, v)$ is the aberrated phase contained in the $i$ th aperture expressed in terms of Zernike polynomial coefficients and $j=\sqrt{-1}$.

The Point Spread Function $(I)$ is the square modulus of the diffracted wave complex amplitude, the latter being given by the Fourier Transform of the incident wave amplitude. The PSF is so expressed by:

$I(x, y)=\widetilde{P}(x, y) \cdot \widetilde{P}^{*}(x, y)$

where $r=\sqrt{x^{2}+y^{2}}, \sim$ denotes the Fourier Transform and $*$ the complex conjugate. The Fourier Transform of the incident aberrated wave amplitude is equal to:

$$
\begin{aligned}
\widetilde{\Psi}=\frac{\pi \cdot f_{\mathrm{c}}^{2}}{2} & {\left[\left(\frac{J_{1}\left(\pi \cdot f_{\mathrm{c}} \cdot r\right)}{\pi \cdot f_{\mathrm{c}} \cdot r} \mathrm{e}^{-j \pi f_{\mathrm{ci}}}\right) \otimes \widetilde{h}_{1}(x, y)+\right.} \\
& \left.\left(\frac{J_{1}\left(\pi \cdot f_{\mathrm{c}} \cdot r\right)}{\pi \cdot f_{\mathrm{c}} \cdot r} \mathrm{e}^{j \pi f_{\mathrm{ci}}}\right) \otimes \widetilde{h}_{2}(x, y)\right]
\end{aligned}
$$


where $J_{1}$ is the first order Bessel function of the first kind. The normalized Optical Transfer Function is given by:

$O(u, v)=\frac{\widetilde{I}(u, v)}{\widetilde{I}(0,0)}$.

As in the ideal case (i.e. no aberrations in the pupil), the OTF consists of three peaks from which the fringe contrast can be evaluated: two high frequency peaks, $O_{\mathrm{HF}}$, containing the coherent energy, and one low frequency peak, $O_{\mathrm{LF}}$, containing the incoherent energy.

The fringe contrast $C$ is defined as the ratio of the coherent to the incoherent energies:

$C=2 \cdot \frac{\left|\int O_{\mathrm{HF}}(u, v) \cdot \mathrm{d} u \cdot \mathrm{d} v\right|}{\left|\int O_{\mathrm{LF}}(u, v) \cdot \mathrm{d} u \cdot \mathrm{d} v\right|}$

$C$, equal to 1 in the ideal case, is in practice degraded by optical aberrations and is always smaller than 1 .

SIVA uses the wavefront output file of CodeV (Zernike polynomial coefficients) to estimate fringe contrast and Airy disk displacement as a function of star position in the field of view. The Airy disk displacement is computed considering the centroid of the point spread function.

SIVA was validated by comparison with $\mathrm{CodeV}$ results in terms of calculation of the Modulation Transfer Function, and real and imaginary parts of the Optical Transfer Function.

\subsection{Sensitivity analysis}

We performed analyses of the Korsch configuration with CodeV to assess its performance in terms of sensitivity to misalignments. This sensitivity is expressed by the centroid displacement of the Airy disk, more severe than visibility criterion, induced by a tilt or decentering of one mirror. This can be considered as relevant enough for these kinds of misalignments, although it is the fringe position that would matter if one wanted to express the tolerances in optical aberrations as piston.

An instantaneous astrometric accuracy of $100 \mu$ as is required in order to achieve the final $10 \mu$ as accuracy on the measurements. Considering the $30-\mathrm{m}$ focal length, this accuracy can be achieved if the centroid displacement induced by spurious effects is less than $15 \mathrm{~nm}$. The misalignments that we analyze here are the followings:

- $\Delta \alpha$ : tilt around the $x$-axis (normal to baseline);

- $\Delta y$ : decentering along the $y$-axis (parallel to baseline);

- $\Delta z$ : decentering along the $z$-axis.

These effects are studied for each aspherical mirror $\left(M_{1}\right.$, $M_{2}$ and $M_{3}$ ). The mirrors $M_{2}$ and $M_{3}$ are monolithic, whereas there are two independent parts of $M_{1}$ for each arm of the interferometer. The contributions of each mirror misalignment to the astrometric accuracy degradation

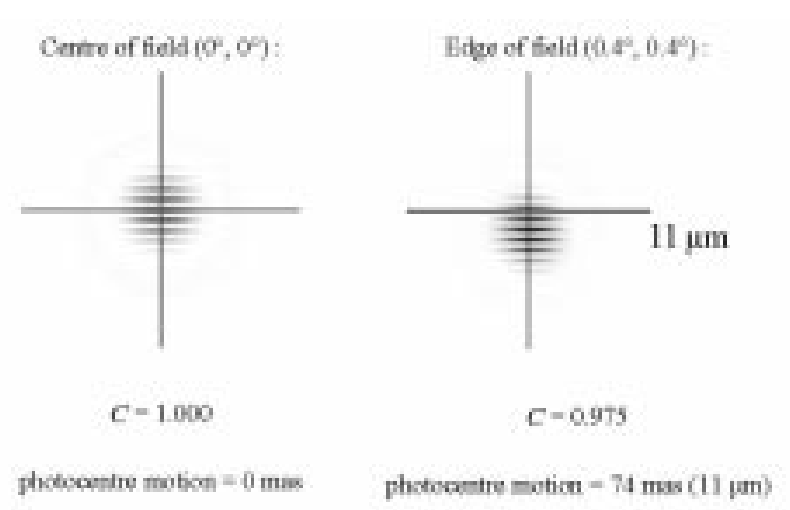

Fig. 5. Visualisation of fringe patterns, contrast and Airy disk centroid displacement computation

Table 3. Contrast and centroid displacement estimated for different points in the field of view

\begin{tabular}{|c|c|c|c|}
\hline $\begin{array}{l}\text { Field point } \\
\text { (deg) }\end{array}$ & $\begin{array}{c}\text { Contrast } \\
(-)\end{array}$ & \multicolumn{2}{|c|}{$\begin{array}{l}\text { Centroid displacement } \\
(\mathrm{mas}) \quad(\mu \mathrm{m})\end{array}$} \\
\hline$(0.0,0.0)$ & 0.998 & 0 & 0 \\
\hline$(0.0,0.2)$ & 0.995 & 30.6 & 4.48 \\
\hline$(0.0,0.4)$ & 0.991 & 20.2 & 3.02 \\
\hline$(0.4,0.4)$ & 0.975 & 74.8 & 11.26 \\
\hline
\end{tabular}

are assumed to be equivalent. The final centroid displacement is assumed to vary as the sum in quadrature of the displacements induced by each contribution. Therefore, each of these contributions must be smaller than $5 \mathrm{~nm}$.

\subsection{Performance results}

\subsubsection{Optical quality}

Figure 5 shows an example of the contrast estimate and Airy disk displacement for two different positions: centre of the field of view and field edge. The hypotheses are:

- pupil diameter: $60 \mathrm{~cm}$;

- baseline: $2.55 \mathrm{~m}$;

- wavelength: $550 \mathrm{~nm}$;

- field of view: $0.8 \times 0.8 \mathrm{deg}^{2}$.

Table 3 shows the estimated monochromatic contrast and Airy disk displacement for different positions in the field of view for the Korsch configuration. The focal length varies in the field due to the optical distortion. The contrast remains high across the whole field $(>95 \%)$. There is a displacement of the centroid, $4.48 \mu \mathrm{m}$ and $11.26 \mu \mathrm{m}$ respectively at the $\left(0.0^{\circ}, 0.2^{\circ}\right)$ and $\left(0.4^{\circ}, 0.4^{\circ}\right)$ points, which has to be taken into account for data calibration.

\subsubsection{Sensitivity to misalignments}

Table 4 gives the estimated maximum tilts and decenterings, for which centroid displacements are maintained 
Table 4. Tolerances of the Korsch configuration for a maximum centroid shift of $5 \mathrm{~nm}$ (34 as)

\begin{tabular}{cc}
\hline & $<5 \mathrm{~nm}$ centroid shift \\
\hline Tilt $\Delta \alpha$ & $20 \mu \mathrm{as}$ \\
$M_{1}$ & $250 \mu \mathrm{as}$ \\
$M_{2}$ & $350 \mu \mathrm{as}$ \\
$M_{3}$ & \\
decentering $\Delta y$ & $1 \mathrm{~nm}$ \\
$M_{1}$ & $1 \mathrm{~nm}$ \\
$M_{2}$ & $3 \mathrm{~nm}$ \\
$M_{3}$ & \\
decentering $\Delta z$ & $2 \mathrm{~nm}$ \\
$M_{1}$ & $3 \mathrm{~nm}$ \\
$M_{2}$ & $40 \mathrm{~nm}$ \\
$M_{3}$
\end{tabular}

smaller than $5 \mathrm{~nm}$. The results show that nanometric metrology is required to control the positions of the mirrors if the astrometric precision must be preserved. The control of the primary mirror $\left(M_{1}\right)$ seems to be the most stringent, as it requires a less than $20 \mu$ as tilt and $1 \mathrm{~nm}$ decentering. The control of the other mirrors is less constraining.

\subsubsection{Distortion}

At the edge of the field $\left(0.4^{\circ}\right)$, the change $\Delta F$ in focal length is $0.06 \mathrm{~m}$, giving a ratio $\Delta F / F$ equal to $2.010^{-3}$. Although this value is 4 times over the requirement defined by Eq. (9), it is not considered as critical as it is possible to overcome it by technical solutions.

\section{Conclusion}

We proposed an optical configuration suitable for a microarcsecond astrometric interferometer in space enabling direct fringe detection. It overcomes the confusion effect present in an optical configuration such as in the initial scheme of GAIA (Lindegren \& Perryman 1996), while considering the expected state-of-the-art CCD technologies and the dimension requirements to fit in the Ariane $\mathrm{V}$ fairing.

We also evaluated the sensitivity of the configuration to misalignments. Results show that tolerances require nanometric metrology and less than 20- $\mu$ as tilt to save the astrometric accuracy. The control of the primary mirror is the most stringent.
We have shown that the state-of-the-art optics and detector technology are at the level of the direct fringe detection requirements with an astrometric mission aiming to reach an accuracy level of $10 \mu \mathrm{as}$. Therefore, it appears that this cornerstone mission could attain, with a less efficient accuracy, the 20 th magnitude, i.e. $10^{9}$ objects.

Acknowledgements. The work described was performed during a study conducted under ALENIA's prime contractorship (in the framework of the APLT-AMTS ESA contract) and the activities were carried out in cooperation with the Observatory of Torino (OATo).

\section{References}

Cecconi M., Gai M., Lattanzi M., 1997a, Battrick B. (ed.), Hipparcos Venice'97, ESA SP-402, p. 803

Cecconi M., Rigoni G., Bernacca P., 1997b, Battrick B. (ed.), Hipparcos Venice'97, ESA SP-402, p. 811

Favata F., Perryman M., 1997, Battrick B. (ed.), Hipparcos Venice'97, ESA SP-402, p. 771

Gai M., Bertinetto F., Bisi M., et al., 1997, Battrick B. (ed.), Hipparcos Venice'97, ESA SP-402, p. 835

Gai M., Lattanzi M., Casertano S., Guarnieri M., 1995, Perryman M. and Leeuwen F.V. (eds.), Future Possibilities For Astrometry in Space, ESA SP-379

Gilmore G., Perryman M.A.C., Lindegren L., et al., 1998, Reasenberg R.D. (ed.), Astronomical Interferometry, Proc. SPIE 3350, p. 541

Kovalevsky J., 1998, ARA\&A 36, 99

Lattanzi M., Spagna A., Sozzetti A., Casertano S., 1997, Battrick B. (ed.), Hipparcos Venice'97, ESA SP-402, p. 755

van Leeuwen F., 1997, Space Sci. Rev. 81, 201

Lindegren L., Perryman M., 1996, A\&A 116, 579

Loiseau S., Shaklan S., 1996, A\&A 117, 167

Mignard F., 1995, Perryman M. and Leeuwen F.V. (eds.), Future Possibilities for Astrometry in Space, ESA SP-379, p. 19

Peek H., Theuwissen A., Kokshoorn A., Daemen E., 1993, IEDM Techn. Digest, p. 567

Peek H., Verbugt D., Beenhakkers M., Huinink W., Kleimann A., 1996, IEDM'96, IEDM Techn. Digest

Perryman M., 1997, Battrick B. (ed.), Hipparcos Venice'97, ESA SP-402, p. 1

Perryman M., Lindegren L., Turon C., 1997, Battrick B. (ed.), Hipparcos Venice'97, ESA SP-402, p. 743

Thomas E., 1996, Evaluation of the Confusion Effect on the GAIA Astrometric Accuracy, Report se/tni 318/96, Aerospatiale

Thomas E., Robbe S., Viard T., et al., 1997, in International Conference on Space Optics ICSO'97, CNES 